\title{
Terapia dual o triple en hipertensión arterial sistémica, ¿a quiénes, cuándo y con qué?
}

\author{
Double or triple combination therapy in systemic arterial hypertension: \\ to whom, when and with what?
}

\author{
Martín Rosas-Peralta, ${ }^{1 *}$ Gabriela Borrayo-Sánchez, ${ }^{1}$ Luis Alcocer, ${ }^{2}$ Juan L. G. Durán-Arenas ${ }^{1}$ y \\ Víctor H. Borja-Aburto ${ }^{1}$ \\ 1'Instituto Mexicano del Seguro Social, Coordinación de Planeación en Salud; ${ }^{2}$ Interamerican Society of Hypertension. Ciudad de México, México
}

La hipertensión arterial sistémica (HAS) es el principal factor de riesgo para muerte prematura en el mundo y en México. ${ }^{1}$ Se estima que existen 15.2 millones de mexicanos con diagnóstico de HAS, de los cuales 7.48 millones son derechohabientes del IMSS (evaluación de los riesgos financieros considerados en el Programa de Administración de Riesgos Institucionales, ENSANUT, 2018). ${ }^{2}$ La HAS es una enfermedad crónica compleja que requiere atención médica continua con estrategias multifactoriales de reducción del riesgo, las cuales van más allá del control numérico en $\mathrm{mm} \mathrm{Hg}$ de la tensión arterial y han demostrado ser efectivas para reducir las complicaciones vasculares, cardiacas y renales de la enfermedad, así como su impacto en la muerte prematura. La educación y el apoyo continuos en materia de autogestión de la HAS son fundamentales para superar los principales retos: el apego terapéutico y la autovigilancia del paciente por el resto de su vida. ${ }^{3,4}$

En la guía de 2017 de la American Heart Association y el American College of Cardiology (AHA/ACC), que actualiza el séptimo informe del Joint National Committee, se propuso reducir el punto de corte para el diagnóstico de HAS a $\geq 130 / 80 \mathrm{~mm} \mathrm{Hg}$, así como iniciar tratamiento farmacológico en los pacientes en estadio 1 (130$140 / 80-90 \mathrm{~mm} \mathrm{Hg}$ ) y con alto riesgo. ${ }^{5}$ Por otra parte, las nuevas recomendaciones de la guía de 2018 de la European Society of Cardiology y la European Society of Hypertension (ESC/ESH) señala que en pacientes con tensión arterial limítrofe o elevada (130-139/80-89 mm Hg) solo debe indicarse modificaciones al estilo de vida, dieta y ejercicio. Estas aparentes divergencias han generado incertidumbre, en especial entre los expertos. ${ }^{6}$ Otro aspecto innovador es la recomendación de usar terapia dual o terapia triple, ya sea de forma separada o en una sola píldora, como tratamiento de primera línea en pacientes con HAS, sobre todo a partir de una tensión arterial $\geq 160 / 100 \mathrm{~mm} \mathrm{Hg} 0 \geq 140 / 90 \mathrm{~mm} \mathrm{Hg}$ si el sujeto es de alto riesgo. ${ }^{6}$

En México, como en la mayoría de los países de Latinoamérica, existen graves rezagos en la detección oportuna de la HAS, así como en su estratificación por riesgo, inicio oportuno del tratamiento, control óptimo, seguimiento del control y de la adherencia terapéutica.

\section{¿A quién dar terapia dual o triple?}

Las directrices más recientes (AHA/ACC y ESC/ $E S H)^{5,6}$ recomiendan iniciar el tratamiento con dos fármacos, de preferencia en una sola píldora, en los pacientes con tensión arterial $\geq 160 / 100 \mathrm{~mm} \mathrm{Hg} 0$ $\geq 140 / 90 \mathrm{~mm} \mathrm{Hg}$ cuando los pacientes están clasificados como de alto riesgo o con daño a órgano blanco (retinopatía, hipertrofia del ventrículo izquierdo, nefropatía, diabetes). A lo anterior hay que agregar que aproximadamente $25 \%$ de los pacientes requerirá tres agentes antihipertensivos para alcanzar las 
metas terapéuticas, las cuales se han tornado más estrictas (< 130/80 mm Hg pero no < 120/70 mm Hg). ${ }^{5,6}$

No estamos de acuerdo del todo con algunos criterios de ACC/AHA establecidos en la guía de 2017:

- El punto de corte en 130/80 mm Hg para determinar la presencia de hipertensión, bajo la premisa de que la mayoría de los pacientes en estadio 1 (130-139/80-89 mm Hg) pueden ser controlados con estrategias no farmacológicas y que únicamente los pacientes con alto riesgo cardiovascular requieren tratamiento farmacológico y solo con un medicamento.

- La tensión arterial < 130/80 mm Hg como meta terapéutica, dado que el contexto en México es muy diferente y que la meta con más beneficio clínico neto (efectividad/efectos colaterales) es el nivel aceptado por las guías europeas de 2018, es decir, $<140 / 90 \mathrm{~mm} \mathrm{Hg}$ y solo si el paciente lo tolera, $<130 / 80 \mathrm{~mm} \mathrm{Hg}$ pero no menos de $120 / 70 \mathrm{~mm} \mathrm{Hg}$.

Estamos de acuerdo con las tendencias de ambas guías de iniciar con esquema farmacológico doble, de preferencia en un solo comprimido, en el entendido de que la mayoría de los pacientes son de riesgo medio-alto, excepto los pacientes de edad avanzada, los frágiles y los jóvenes de alto riesgo con tensión arterial > 130/80 mm Hg, en quienes estaría indicada la monoterapia.

\section{¿Con qué? Terapia dual y terapia triple}

El efecto simultáneo del sistema renina-angiotensina-aldosterona, sistema nervioso autónomo, reactividad vascular y función endotelial, entre otros, pone de manifiesto que el bloqueo de un solo mecanismo puede no resultar suficiente, por lo que la monoterapia resulta insuficiente, en tanto que la combinación de dos agentes antihipertensivos con un mecanismo de acción diferente puede reducir los eventos coronarios hasta en aproximadamente $35 \%$ y los eventos cerebrovasculares en $54 \%{ }^{7}$

Si existe suficiente evidencia de los aspectos fisiopatológicos múltiples, ¿por qué predomina la prescripción de monoterapia? La razón más expresada por el médico general es el temor de bajar la presión arterial de forma súbita y extrema. Sin embargo, cada vez existe más evidencia de que con la combinación de fármacos se alcanzan cifras de control con mayor consistencia y sin efectos colaterales. Un calcioantagonista $(\mathrm{CaA})$ con un diurético o un bloqueador del receptor de angiotensina (ARA2) o un inhibidor de la enzima convertidora de angiotensina (IECA) con un diurético, o un IECA o un ARA2 con $\mathrm{CaA}$ son las combinaciones popularizadas como "terapia dual antihipertensiva", las cuales incluso ya existen en una sola píldora, lo que facilita su prescripción y la adherencia terapéutica por parte del paciente. ${ }^{8}$

El uso de un betabloqueador con un diurético también es terapia dual, sin embargo, solo se recomienda en ciertos pacientes (con isquemia, taquiarritmia o insuficiencia cardiaca). Hay combinaciones que no deben emplearse, como dos agentes que inhiben el eje renina-angiotensina (el 20 de abril de 2012, la Food and Drug Administration publicó una advertencia al respecto), específicamente IECA con ARA2. ${ }^{9}$ Se ha observado que las combinaciones de diuréticos con betabloqueantes tienden a incrementar el riesgo de desarrollar diabetes tipo 2, como se registró en el ensayo ASCOT, ${ }^{10}$ por lo que deben utilizarse con cautela, sobre todo en población diabetogénica como la mexicana. Tampoco se recomienda la combinación de bloqueadores betaadrenérgicos con antagonistas del calcio no dihidropiridínicos, por el mayor riesgo de bradicardia o bloqueo auriculoventricular. ${ }^{11}$ Los pasos del tratamiento de los pacientes con tensión arterial $>$ 150/100 mm Hg según la guía ESC/ESH de 2018 se muestran en la Figura 1.

La monoterapia solo debe ser intentada en los sujetos con HAS leve (140-149/90-99 mm Hg) y bajo riesgo cardiovascular o en casos especiales, como en el paciente frágil o bien en la HAS gestacional. ${ }^{12-16}$

\section{¿Cuándo preferir terapia dual con diurético y cuándo con calcioantagonista?}

En los pacientes con obesidad o tendencia a problemas metabólicos es preferible la combinación de IECA o ARA2 con CaA dihidropiridínico (amlodipino, nicardipino, felodipino); si no hay obesidad ni problemas metabólicos pero existe evidencia de retención hídrica es preferible la combinación de un IECA o ARA2 con un diurético tiazídico si la función renal está conservada (velocidad de filtración glomerular $>40 \mathrm{~mL} /$ minuto); o bien, un diurético de asa si la función renal está deteriorada $\left(<30 \mathrm{~mL} /\right.$ minuto). ${ }^{11-16}$

En el estudio ACCOMPLISH se incluyeron 11462 pacientes con alto riesgo cardiovascular mayores de 55 años (60.4 \% con diabetes mellitus); se dividieron en dos grupos: un grupo recibió la combinación de benazepril más amlodipino y el otro, benazepril más 


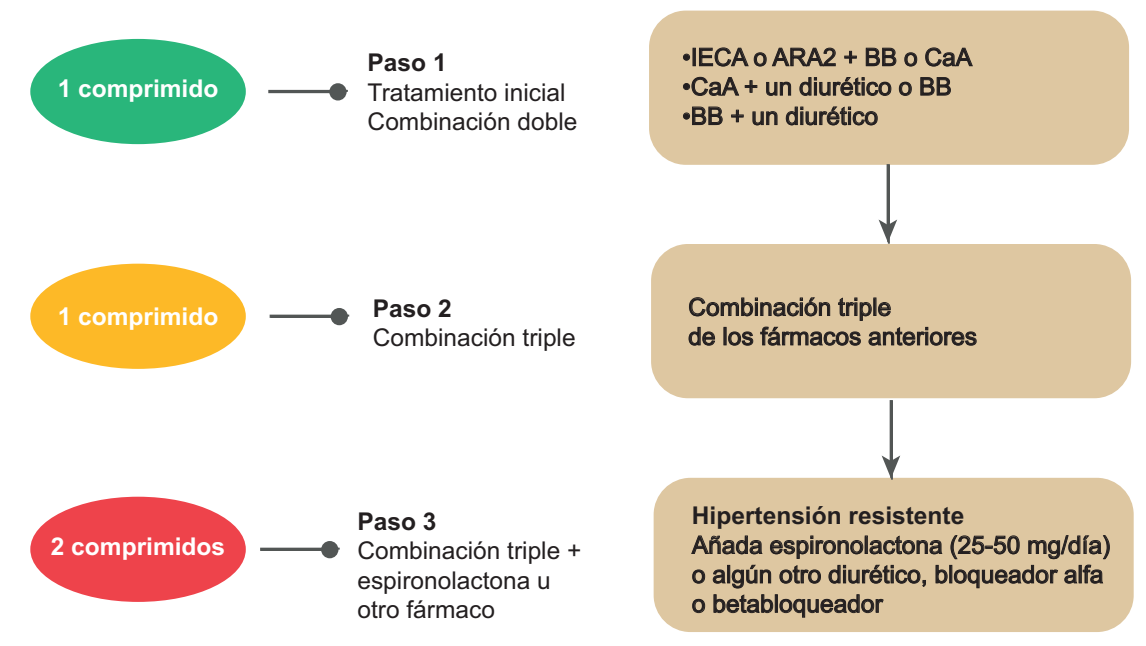

Figura 1. Propuesta en pasos para el tratamiento del paciente de alto riesgo portador de hipertensión arterial sistémica en estadios 1,2 y 3. IECA = inhibidor de la enzima convertidora de angiotensina, ARA2 = antagonista del receptor de angiotensina 2, BB = betabloqueador, $\mathrm{Ca} A=$ calcioantagonista dihidropiridínico. Adaptación de las guías europeas (ESC/ESH). ${ }^{6}$

hidroclorotiazida. La duración del ensayo estaba programada a cinco años, sin embargo, el estudio se suspendió en el mes 39 porque el antagonista del calcio en combinación con IECA con más eficaz que la combinación de IECA más hidroclorotiazida en la reducción de eventos cardiovasculares, cerebrovasculares y renales. ${ }^{16}$

\section{Conclusión}

Al bloquear varias vías de aumento de la presión arterial, la terapia combinada tiene mayor poder antihipertensivo que la monoterapia en dosis altas, además de proporcionar mayor protección a los órganos diana y tener menor potencial de efectos secundarios.

Las combinaciones más utilizadas incluyen un IECA ○ ARA2 asociado a un antagonista del calcio o un natriurético. Las combinaciones que incluyen un diurético han dado mejores resultados en pacientes con insuficiencia cardiaca o deterioro de la función renal.

Un porcentaje significativo de pacientes requerirá terapia triple: un IECA o ARA2, un CaA y un natriurético, la cual debe administrarse a los pacientes que no respondan al tratamiento dual en seis a ocho semanas, ya que el beneficio está fuera de toda duda. El empleo de estas combinaciones en forma de un solo comprimido tiene un impacto muy favorable en la adherencia terapéutica por parte del paciente.

\section{Conflicto de intereses}

Ninguno.

\section{Financiamiento}

La presente investigación no ha recibido ninguna beca específica de agencias de los sectores público, comercial, o sin ánimo de lucro.

\section{Responsabilidades éticas}

Los autores declaran que para esta investigación no se han realizado experimentos en seres humanos ni en animales.

Confidencialidad de los datos. Los autores declaran que en este artículo no aparecen datos de pacientes.

Derecho a la privacidad y consentimiento informado. Los autores declaran que en este artículo no aparecen datos de pacientes.

\section{Bibliografía}

1. Rosas-Peralta M, Borrayo-Sánchez G, Santiago-López J, Ramírez-Arias E, García-Méndez R, Borja-Aburto VH, et al. What is new in hypertension of Mexico 2018? - Impact of the new classification of high blood pressure in adults from American College of Cardiology/American Heart Association (ACC/AHA). Ann Clin Hypertens. 2018;2:24-30.

2. Secretaría de Salud, Instituto Nacional de Salud Pública, Instituto Nacional de Estadística y Geografía. Encuesta Nacional de Salud y Nutrición 2018. México: SSa, INSP, INEGI; 2019.

3. Oparil S, Acelajado MC, Bakris GL, Berlowitz DR, Cífková R, Dominiczak AF, et al. Hypertension. Nat Rev Dis Primers. 2018 Mar 22;4:18014.

4. Sánchez RA, Ayala M, Baglivo $H$, Velázquez C, Burlando G, Kohlmann O, et al. Latin American guidelines on hypertension. Latin American Expert Group. J Hypertens. 2009;27:905-922. 
5. Whelton PK, Carey RM, Aronow WS, Casey DE, Collins KJ, Dennison-Himmelfarb C, et al. 2017 ACC/AHA/AAPA/ABC/ACPM/AGS/APhA ASH/ASPC/NMA/PCNA Guideline for the Prevention, Detection, Evaluation, and Management of High Blood Pressure in Adults: a report of the American College of Cardiology/American Heart Association Task Force on Clinical Practice Guidelines. Hypertension. 2017;71:1269-1324.

6. Williams B, Mania G, Spiering W, Agabiti-Rosei E, Azizi M, Burnier M, et al. 2018 ESC/ESH Guidelines for the Management of Arterial Hypertension: the Task Force for the Management of Arterial Hypertension of the European Society of Cardiology and the European Society of Hypertension. J Hypertens. 2018;36:1953-2041.

7. Wald DS, Law M, Morris JK, Bestwick JP, Wald NJ. Combination therapy versus monotherapy in reducing blood pressure: meta-analysis on 11,000 participants from 42 trials. Am J Med. 2009;122:290-300.

8. Pimenta E, Oparil S. Fixed combinations in the management of hypertension: patient perspectives and rationale for development and utility of the olmesartan-amlodipine combination. Vasc Health Risk Manag. 2008;4:653-664.

9. Burnier M. Antihypertensive combination treatment: state of the art. Curr Hypertens Rep. 2015;17:51.

10. Poulter NR, Wedel H, Dahlöf B, Sever PS, Beevers DG, Caulfield M, et al. Role of blood pressure and other variables in the differential cardiovascular event rates noted in the Anglo-Scandinavian Cardiac Outcomes Trial-Blood Pressure Lowering Arm (ASCOT-BPLA). Lancet. 2005;366:907-913.
11. Rubio-Guerra AF, Castro-Serna D, Elizalde-Barrera Cl, Ramos-Brizuela LM. Current concepts in combination therapy for the treatment of hypertension: combined calcium channel blockers and RAAS inhibitors. Integr Blood Press Control. 2009;2:55-62.

12. Bakris GL. Combined therapy with a calcium channel blocker and an angiotensin II type 1 receptor blocker. J Clin Hypertens. 2008;10:27-32.

13. James PA, Oparil S, Carter BL, Cushman WC, Dennison-Himmelfarb C, Handler J, et al. 2014 evidence-based guideline for the management of high blood pressure in adults: report from the Panel Members Appointed to the Eighth Joint National Committee (JNC 8). JAMA. 2014;311:507-520.

14. Task Force for the Management of Arterial Hypertension of the European Society of Hypertension, Task Force for the Management of Arterial Hypertension of the European Society of Cardiology. 2013 ESH/ESC guidelines for the management of arterial hypertension. Blood Press. 2013:22:193-278.

15. Law MR, Morris JK, Wald NJ. Use of blood pressure lowering drugs in the prevention of cardiovascular disease: meta-analysis of 147 randomized trials in the context of expectations from prospective epidemiological studies. BMJ. 2009;338:b1665

16. Jamerson K, Weber MA, Bakris GL, Dahlof B, Pitt B, Shi V, et al. Benazepril plus amlodipine or hydrochlorothiazide for hypertension in high-risk patients. N Engl J Med. 2008;359:2417-2428. 